\title{
The need for integrative computational oncology: an illustrated example through MMP-mediated tissue degradation
}

\author{
Shannon M. Mumenthaler ${ }^{1}$, Gianluca D'Antonio' ${ }^{2}$, Luigi Preziosi ${ }^{2}$ and Paul Macklin ${ }^{1,3}$ * \\ ${ }^{1}$ Center for Applied Molecular Medicine, Keck School of Medicine, University of Southern California, Los Angeles, CA, USA \\ 2 Dipartimento di Scienze Matematiche, Politecnico di Torino, Torino, Italia \\ ${ }^{3}$ Department of Biomedical Engineering, Viterbi School of Engineering, University of Southern California, Los Angeles, CA, USA
}

\section{Edited by:}

Katarzyna Anna Rejniak, H. Lee Moffitt Cancer Center \& Research Institute, USA

\section{Reviewed by:}

Agnès Noël, University of Liege, Belgium

Georgios Lolas, National Technical University of Athens, Greece

*Correspondence:

Paul Macklin, Center for Applied Molecular Medicine, Keck School of

Medicine, University of Southern

California, 2250 Alcazar Street, Suite

242, Los Angeles, CA 90033, USA

e-mail: paul.macklin@usc.edu

\begin{abstract}
Physical oncology is a growing force in cancer research, and it is enhanced by integrative computational oncology: the fusion of novel experiments with mathematical and computational modeling. Computational models must be assessed with accurate numerical methods on correctly scaled tissues to avoid numerical artifacts that can cloud analysis. Simulation-driven analyses can only be validated by careful experiments. In this perspectives piece, we evaluate a current, widespread model of matrix metalloproteinasedriven tissue degradation during cancer invasion to illustrate that integrative computational oncology may not realize its fullest potential if either of these critical steps is neglected.
\end{abstract}

Keywords: matrix metalloproteinase, tissue degradation, integrative modeling, cancer, computational oncology

\section{INTRODUCTION}

Physical oncology - the study of the physical biology of cancer, the development of new physical measurement platforms, and the use of mathematical and computational modeling to understand complex cancer systems - has emerged as an important force in cancer research (1). Key to this approach is integrative computational oncology: multidisciplinary teams of biologists, oncologists, physicists, engineers, and mathematicians working together to generate novel platforms, where modeling informs experiments, and experiments drive modeling. Mathematical modeling can describe and simplify complex systems, facilitating analysis. Accurate simulations assist the analysis of these systems, yielding observations that drive biological hypotheses. Experimental biology is necessary for validating and refining these hypotheses and advancing our understanding of cancer. This special issue discusses successful examples of applying integrative modeling to cancer-related questions. However, neglecting any of these key ingredients can be detrimental and may blind teams to subtle modeling flaws, potentially resulting in misleading model assessment, incorrect biological conclusions, or unverifiable predictions.

In this perspectives piece, we will look at a widely used mathematical model of tissue degradation by matrix metalloproteinases (MMPs) in order to illustrate (1) the need for evaluation of mathematical models by proper numerical techniques, applied to biologically relevant space and time scales, and (2) that even with proper numerical analysis, only experiments can truly validate mathematical model predictions and help choose among plausible explanations of model findings.

\section{MMP-MEDIATED TISSUE DEGRADATION}

Progression from in situ carcinoma - where growth is constrained to a local site by a fully intact basement membrane (BM) - to invasive carcinoma requires disruption of the $\mathrm{BM}$ and penetration into the surrounding stroma. Once in the stroma, invading cancer cells often degrade and remodel the extracellular matrix (ECM) and later break through BM to enter blood vessels - a key step in metastasis. A quantitative understanding of proteolytic degradation of tissue is necessary in predicting (and disrupting!) cancer invasion and metastasis. It is currently unclear whether tissue degradation is primarily due to MMPs secreted by cancer cells or by stromal cells in response to tumor signaling. Quantitative modeling could help narrow down the possibilities to the most plausible models of stromal invasion, which can then be experimentally tested and validated.

Extracellular matrix is a 3-D cross-linked network of proteins and polysaccharides that provides structural support to cells; BM is a specialized form of ECM, although thinner (50$100 \mathrm{~nm}$ ) and more dense $(2,3)$. ECM (including BM) can be degraded by MMPs secreted by tumor, stromal, and immune cells (4-6). MMPs are secreted in an inactive form that must be cleaved into an active form, and are further regulated by inhibitors of metalloproteinases. MMPs may be soluble and diffuse through tissue (e.g., MMP9), or membrane-bound (e.g., MTMMP1) (6).

The most widely used tissue degradation models focus on soluble MMPs using reaction-diffusion equations [e.g., (7-9)], neglect inhibitors and promoters, and assume the MMP is immediately active. If $E$ is the ECM density (or volume fraction) and $M$ is the 
MMP concentration (both dimensionless), then

$$
\begin{aligned}
\frac{\partial M}{\partial t} & =\nabla \cdot(D \nabla M)+s(\mathbf{X}, t)-r_{M} E M-d_{M} M \\
\frac{\partial E}{\partial t} & =-r_{E} E M .
\end{aligned}
$$

$D$ is the diffusion constant, $s$ is the source (tumor or other cells), $r_{M}$ and $r_{E}$ are reaction rates, and $d_{M}$ is the MMP decay rate. These standard reaction-diffusion equations are typically solved with finite differences on a Cartesian mesh [e.g., (7-9)]. The most clinically oriented BM degradation model we know of simulated BM as denser ECM on the same ECM computational mesh (10).

\section{FUNCTIONAL FORMS AND PARAMETER VALUES}

For this discussion, we assume $D=D_{0}(1-E)$ for a constant $D_{0}$. We set $D_{0}=8 \times 10^{-9} \mathrm{~cm}^{2} / \mathrm{s}$ (11), $r_{M}=r_{E}=1 / 200 \mathrm{~s}^{-1}$ (11), and $d_{M}=5 \times 10^{-5} \mathrm{~s}^{-1}(12)$. This gives an (ECM-dependent) reactiondiffusion length scale $L$ of $\sqrt{D_{0}(1-E) /\left(r_{M} E+d_{M}\right)} \sim 10 \mu \mathrm{m}$ (" $\sim$ "denotes "on the order of") for $0 \leq E \leq 0.90$. This matches our biophysical expectations: MMPs are relatively large macromolecules diffusing through a tortuous ECM structure, so the length scale should be significantly smaller than for oxygen (typically $\sim 100 \mu \mathrm{m})$. We initially set the $E=0.85$.

\section{MODEL EVALUATION REQUIRES GOOD NUMERICAL TECHNIQUES}

Accurate numerical solution (and hence proper evaluation) of the model and its $\sim 10 \mu \mathrm{m}$ length scale requires an $\sim 1 \mu \mathrm{m}$ mesh size. To date, most published work has used small diffusion constants on relatively large 10-20 $\mu \mathrm{m}$ meshes [e.g., $(7,8)]$. We solve Eqs 1 and 2 on a $1 \mu \mathrm{m}$ mesh with standard centered finite differences, using the ghost fluid method to implement boundary conditions wherever the computational stencil intersects the BM (zero flux, or Neumann condition) or a cell boundary (constant, or Dirichlet condition for secreting cells; Neumann condition for non-secreting cells) (13-15). We describe the BM position as in D'Antonio et al. (16). Tumor cell sizes are set to the values in Macklin et al. (17).

\section{CURRENT MODELS PREDICT RAPID "TUNNELING" THROUGH ECM}

We simulated MMP secretion by stromal cells, as one might expect in response to tumor-secreted pro-inflammatory signals. To simplify the analysis, we set $M=1$ on the stromal cells and positioned them at a fixed $10 \mu \mathrm{m}$ from the BM (Figure 1: top left).

In the simulations, MMPs etch out a "hole" in the ECM whose edge expands outward at $\sim 1 \mu \mathrm{m}$ per minute. See Figure 1 for the ECM distribution at 15, 25, and $40 \mathrm{~min}$. This is consistent with an order of magnitude estimate using a Fisher-Kolmogorov-type traveling wave front speed:

$$
\nu=2 L(E) r_{M}(E) \sim 2 \sqrt{D_{0}(1-E) E r_{M}}
$$

gives speeds of $0-3.75 \mu \mathrm{m} / \mathrm{min} \sim 1 \mu \mathrm{m} / \mathrm{min}$ for $0 \leq E \leq 0.90$, where $L(E)$ is as above and $r_{M}(E)=r_{M} E$.

A $1 \mu \mathrm{m} / \mathrm{min}$ expansion rate of the degraded region is comparable to experimentally measured motile tumor cell velocities [e.g., $58.56 \pm 1.62 \mu \mathrm{m} / \mathrm{h}$ for neuN cells in (7)]. The predicted expansion speed is quantitatively consistent with localized ECM degradation "keeping pace" with motile cells as they "tunnel" through the ECM. The simple ECM-MMP model (with sufficient numerical resolution) can produce biologically reasonable results on small time and spatial scales.

However, if extrapolated over long times, this model predicts that a $10 \mathrm{~cm}$ diameter of tissue could be degraded in about a month! This outpaces typical tumor expansion rates by over an order of magnitude: brain tumors (among the fastest growing tumors) typically expand at $80-100 \mu \mathrm{m} /$ day (18), requiring at least 500 days to infiltrate a $10 \mathrm{~cm}$ tissue. The simple MMP model would therefore predict an ever-widening gap between the advancing tumor front and the edge of the degraded tissue, contradicting typical observations that MMP activity is localized near the boundary of an advancing tumor.

This widely used model, once simulated accurately, does not adequately describe MMP-mediated tissue degradation around growing tumors. Neglected factors (e.g., activators and inhibitors) may be needed to confine proteolytic activity near tumor boundaries; similar approaches have been used to model urokinase-type plasminogen activators in tissue degradation (19). Alternatively, non-diffusing membrane-bound MMPs may be more relevant. New imaging technologies that dynamically capture ECM degradation could help select among possible alternative models (20, 21). Recent integrative experimental-computational work showed the critical role of MT-MMP activity during cancer cell invasion, finding that MT1-MMP turnover could be a potent anti-invasion therapeutic target (22). Ultimately, only carefully planned and executed experiments can help choose between these and other possible explanations.

\section{ASSESSING DEGRADATION OF THE BASEMENT MEMBRANE}

A $100 \mathrm{~nm}$ BM cannot be properly resolved on a $1 \mu \mathrm{m}$ mesh. Solving (1)-(2) by finite differences (with correct physical dimensions) requires a prohibitive $10 \mathrm{~nm}$ computational mesh. Some have investigated this problem by solving on non-physiological basement membranes [e.g., one cellular automaton mesh point, or $10 \mu \mathrm{m}$ thick (10)], making it difficult to evaluate the models.

Let us instead analyze a simplified problem to estimate the time scale to degrade a BM. Consider a small piece of BM of crosssectional area $A$, volume fraction $F$, and thickness $T(t)$. The total amount of matrix $E(t)$ in the BM section is $A F T(t)$. If $\mathrm{BM}$ is degraded as in (2), then $\mathrm{d} E / \mathrm{d} t=-r_{E} M(t) E(t)$. If $M$ is constant, then the time $t_{\mathrm{B}}$ required to degrade the $\mathrm{BM}$ to some threshold breaking amount $E_{\mathrm{B}}$ is given by

$$
t_{B}=-\ln \left(E(0) / E_{B}\right) / r_{B} M .
$$

If $r_{\mathrm{E}}=1 / 200 \mathrm{~s}^{-1}$ and $M=1$, then a $100 \mathrm{~nm}$ section of $\mathrm{BM}$ is reduced to $10 \mathrm{~nm}$ thick $\left[E_{\mathrm{B}} / E(0)=0.1\right]$ in under $8 \mathrm{~min}$, and to $1 \mathrm{~nm}$ thick $\left(E_{\mathrm{B}} / E(0)=0.01\right)$ in about $15 \mathrm{~min}$.

This suggests several possibilities. (1) The cell "decision" of when to secrete MMPs is the limiting factor to penetrating the $\mathrm{BM}$, rather than the proteolytic process itself. (2) Additional, nonmodeled promoters/inhibitors are rate limiting. Only follow-up experiments can help determine the most plausible explanation, but rapid penetration of the BM by "willing" cells seems consistent with Boyden transwell migration assays (23) and known rapid 




FIGURE 1 | Top left: initial configuration of epithelium (white lumen and tumor cells), a $100 \mathrm{~nm}$ basement membrane, stroma (orange), and stromal cells (red) that secrete MMPs. Remaining plots: ECM volume fraction [ranging from blue $(0 \%)$ to red $(85 \%)$ ] at 15,25 , and 40 min using a widespread ECM-MMP model with a biophysically reasonable reaction-diffusion length scale $(\sim 10 \mu \mathrm{m})$ and degradation rate $\left(\sim 0.1-1 \mathrm{~min}^{-1}\right)$.

( minutes) transmigration of leukocytes through endothelial and epithelial layers and associated membranes $(24,25)$.

\section{CLOSING THOUGHTS}

Accurate models are needed to simplify, analyze, and assess complex phenomena observed in cancer biology. In order to truly assess a model's underlying assumptions, evaluate its predictive value, and study its potential clinical utility, one must use proper numerical methods, reasonable geometries, and experimental validation. As illustrated by the tissue degradation model above, neglecting any of these key factors can lead to inaccurate dynamics, and may potentially cause a team to prematurely accept biological hypotheses. Dynamic feedback between experimental and computational biology systems is necessary to

\section{REFERENCES}

1. Agus DB, Michor F. The sciences converge to fight cancer. Nat Phys (2012) 8:773-4. doi:10.1038/ nphys 2464

2. Leblond CP, Inoue S. Structure, composition, and assembly of basement membrane. Am J Anat (1989) 185:367-90. doi:10.1002/aja. 1001850403

3. Liddington RC. Mapping out the basement membrane. Nat Struct Mol Biol (2001) 8:573-4. doi:10. $1038 / 89590$

4. Coussens LM, Werb Z. Matrix metalloproteinases and the development of cancer. Chem Biol (1996) 3:895-904. doi:10.1016/ S1074-5521(96)90178-7

5. Egeblad M, Werb Z. New functions for the matrix metalloproteinases in cancer progression. Nat Rev Cancer (2002) 2:161-74. doi:10.1038/ nrc745

6. Kessenbrock K, Plaks V, Werb Z. Matrix metalloproteinases: regulators of the tumor microenvironment. Cell (2010) 141:52-67. doi: 10.1016/j.cell.2010.03.015

7. Anderson AR, Hassanein M, Branch KM, Lu J, Lobdell NA, Maier J, et al. Microenvironmental independence associated with tumor progression. Cancer Res (2009) 69:8797-806. doi:10.1158/ 0008-5472.CAN-09-0437

8. Macklin P, McDougall SR, Anderson ARA, Chaplain MAJ, Cristini $\mathrm{V}$, Lowengrub JS. Multiscale modelling and nonlinear simulation of vascular tumour growth. J Math Biol (2009) 58:765-98. doi:10.1007/ s00285-008-0216-9

9. Martin NK, Gaffney EA, Gatenby RA, Maini PK. Tumour-stromal interactions in acid-mediated invasion: a mathematical model. J Theor Biol (2010) 267:461-70. doi:10. 1016/j.jtbi.2010.08.028

10. Basanta D, Strand DW, Lukner RB, Franco OE, Cliffel DE, Ayala $\mathrm{GE}$, et al. The role of transforming growth factor-beta-mediated tumor-stroma interactions in prostate cancer progression: an integrative approach. Cancer Res (2009) 69:7111-20. doi:10.1158/ 0008-5472.CAN-08-3957

11. Saffarian S, Collier IE, Marmer BL, Elson EL, Goldberg G. Interstitial collagenase is a Brownian ratchet driven by proteolysis of collagen. Science (2004) 306:108-11. doi:10. $1126 /$ science. 1099179

drive and improve model development and refinement while ensuring that any resulting integrative platform is clinically relevant.

\section{ACKNOWLEDGMENTS}

Paul Macklin and Shannon M. Mumenthaler thank the USC Center for Applied Molecular Medicine for generous computational resources, and the National Institutes of Health for the Physical Sciences Oncology Center grant 5U54CA143907 for Multiscale Complex Systems Transdisciplinary Analysis of Response to Therapy (MC-START). Paul Macklin thanks the USC James H. Zumberge Research and Innovation Fund (2012 Large Interdisciplinary Award) for support via the Consortium for Integrative Computational Oncology.

12. Kim Y, Friedman A. Interaction of tumor with its micro-environment: a mathematical model. Bull Math Biol (2010) 72:1029-68. doi:10. 1007/s11538-009-9481-z

13. Macklin P, Lowengrub JS. Evolving interfaces via gradients of geometry-dependent interior Poisson problems: application to tumor growth. J Comput Phys (2005) 203:191-220. doi:10.1016/j.jcp.2004.08.010

14. Macklin P, Lowengrub JS. Nonlinear simulation of the effect of microenvironment on tumor growth. $J$ Theor Biol (2007) 245:677-704. doi: 10.1016/j.jtbi.2006.12.004

15. Macklin P, Lowengrub JS. A new ghost cell/level set method for moving boundary problems: application to tumor growth. J Sci Comput (2008) 35:266-99. doi:10.1007/ s10915-008-9190-z

16. D’Antonio G, Macklin P, Preziosi L. An agent-based model for elasto-plastic mechanical interactions between cells, basement membrane and extracellular matrix. Math Biosci Eng (2013) 10:75-101. doi:10.3934/mbe.2013.10.75

17. Macklin P, Edgerton ME, Thompson AM, Cristini V.
Patient-calibrated agent-based modelling of ductal carcinoma in situ (DCIS): from microscopic measurements to macroscopic predictions of clinical progression. J Theor Biol (2012) 301:122-40. doi:10.1016/j.jtbi.2012.02.002

18. Massey SC, Assanah MC, Lopez KA, Canoll P, Swanson KR. Glial progenitor cell recruitment drives aggressive glioma growth: mathematical and experimental modelling. $J \quad R$ Soc Interface (2012) 9:1757-66. doi:10.1098/rsif.2012.0030

19. Chaplain MAJ, Lolas G. Mathematical modelling of cancer cell invasion of tissue: the role of the urokinase plasminogen activation system. Math Models Methods Appl Sci (2005) 15:1685-734. doi:10.1142/S0218202505000947

20. Littlepage LE, Sternlicht $\mathrm{MD}$, Rougier N, Phillips J, Gallo E, Yu Y, et al. Matrix metalloproteinases contribute distinct roles in neuroendocrine prostate carcinogenesis, metastasis, and angiogenesis progression. Cancer Res (2010) 70:2224-34. doi:10.1158/0008-5472.CAN-093515 
21. Scherer RL, VanSaun MN, McIntyre JO, Matrisian LM. Optical imaging of matrix metalloproteinase-7 activity in vivo using a proteolytic nanobeacon. Mol Imaging (2008) 7:118-31.

22. Hoshino D, Koshikawa N, Suzuki T, Quaranta V, Weaver AM, Seiki M, et al. Establishment and validation of computational model for MT1MMP dependent ECM degradation and intervention strategies. PLoS Comput Biol (2012) 8:e1002479. doi:10.1371/journal.pcbi.1002479

23. Albini A, Iwamoto Y, Kleinman HK, Martin GR, Aaronson SA, Kozlowski
JM, et al. A rapid in vitro assay for quantitating the invasive potential of tumor cells. Cancer Res (1987) 47:3239-45.

24. Ginzberg HH, Cherapanov V, Dong Q, Cantin A, McCulloch CA, Shannon PT, et al. Neutrophil-mediated epithelial injury during transmigration: role of elastase. Am J Physiol Gastrointest Liver Physiol (2001) 281:G705-17.

25. Shaw SK, Bamba PS, Perkins BN, Luscinskas FW. Real-time imaging of vascular endothelial-cadherin during leukocyte transmigration across endothelium. I Immunol (2001) 167:2323-30.

Conflict of Interest Statement: The authors declare that the research was conducted in the absence of any commercial or financial relationships that could be construed as a potential conflict of interest.

Received: 15 February 2013; accepted: 11 July 2013; published online: 26 July 2013 Citation: Mumenthaler SM, D'Antonio $G$, Preziosi L and Macklin P (2013) The need for integrative computational oncology: an illustrated example through MMP-mediated tissue degradation. Front. Oncol. 3:194. doi 10.3389/fonc.2013.00194

This article was submitted to Frontiers in Molecular and Cellular Oncology, a specialty of Frontiers in Oncology.

Copyright (c) 2013 Mumenthaler, D'Antonio, Preziosi and Macklin. This is an open-access article distributed under the terms of the Creative Commons Attribution License, which permits use, distribution and reproduction in other forums, provided the original authors and source are credited and subject to any copyright notices concerning any third-party graphics etc. 